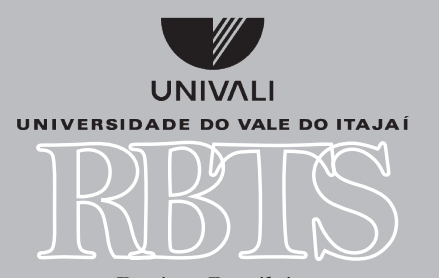

Revista Brasileira de Tecnologias Sociais
1 Profissional de Educação Física. Especialista em Saúde da Família - FURB e Educação Infantil, Séries Iniciais e Gestão Escolar IPEGEX. Mestranda em Saúde e Gestão do Trabalho - UNIVALI. E-mail: dessa.efi@gmail.com.

2 Professora do Programa do Mestrado em Saúde e Gestão do Trabalho - UNIVALI. E-mail: asouza@univali.br.
Revista Brasileira de Teonologias Sociais, v.1, n.2, 2014

doi: $10.1421 / 6 /$ rbts. y1.n2.p27-34

Artigo\$

\section{Protocolo de atendimento do núcleo de apoio à saúde da família do município de Itapema SC: uma contribuição para o processo de trabalho}

\section{Care protocol of the family health support unit of the municipality of Itapema SC: a contribution to the work process}

\author{
Andressa Alves ${ }^{1}$; Antônia Egidia de Souza ${ }^{2}$
}

Resumo: A proposta desse estudo consiste em apresentar a criação do Protocolo de Atendimento para a Equipe do Núcleo de Apoio à Saúde da Família como um instrumento de organização do processo de trabalho. A decisão da criação do protocolo de atendimento surgiu na reunião de equipe NASF em 2012. Sua elaboração teórica foi iniciada em 2013 pelas profissionais de Educação Física e Nutrição, para posterior contribuição das profissionais de Psicologia, Fisioterapia, Fonoaudiologia e Farmácia. O Protocolo de Atendimento do Núcleo de Apoio à Saúde da Família (PANASF) de Itapema (SC) foi elaborado devido à necessidade percebida pelas profissionais que o compõem, necessidade esta advinda da importância de ter respaldo nas atuações e de organizar o processo de trabalho e a rede de atendimento. Visou-se qualificar o serviço e evitar que a política de governo impere sobre as atuações da equipe. Por meio do PANASF objetivou-se ainda uma maior divulgação da composição da equipe NASF Itapema e facilitar a compreensão dos profissionais de saúde e população em geral acerca do que é o Núcleo de Apoio à Saúde da Família, como solicitar/acessar este apoio e quais os serviços prestados pela equipe NASF.

Palavras-chave: NASF. Protocolo de Atendimento. Profissional de Educação Física.

Abstract: The purpose of this study is to present the creation of the Care Protocol for the Family Health Support Unit team of the Family as a tool for organizing the work process. The decision to create the care protocol was made at a meeting of the NASF team in 2012. The theoretical work was started in 2013 by professionals of Physical Education and Nutrition, with subsequent contributions by professional psychologists, Physical Therapists, Speech Therapists and Pharmacists. The Care Protocol of the Family Health Support Unit (PANASF) of Itapema (SC) was developed due to a perceived need among the professionals that comprise it, based on the importance of having support for the actions, and to organize the work process and the care network. The aim was to qualify the service and prevent the government policy prevailing over the actions of the team. Other aims of the PANASF were to create wider dissemination of the composition of the NASF Itapema team, and facilitate understanding among health professionals and the general public, about the work of the Family Health Support Unit, how to request/access this support, and what services are provided by the NASF team.

Keywords: NASF. Care protocol. Physical Education Professional. 


\section{INTRODUÇÃO}

Neste estudo, apresenta-se o processo de criação, aperfeiçoamento e aprovação do Protocolo de Atendimento do Núcleo de Apoio à Saúde da Família de Itapema - SC.

O Núcleo de Apoio à Saúde da Família (NASF) são equipes multiprofissionais que devem atuar de maneira integrada e apoiando os profissionais das equipes de Saúde da Família e das equipes de Atenção Básica, sendo assim o apoio especializado na atenção básica. Dentre outros objetivos, o NASF deve privilegiar a construção de redes de atenção e cuidado, compartilhar práticas e saberes em saúde com as equipes de referência apoiadas, auxiliar no manejo e/ou resolução de problemas clínicos e sanitários, bem como ampliar o escopo de ofertas na atenção básica ao agregar práticas, buscando superar a fragmentação (BRASIL, 2014).

Esse protocolo foi construído pelos profissionais que compõem a equipe do Núcleo de Apoio à Saúde da Família de Itapema, por anseio em organizar o serviço e fazer com que este seja conhecido e acessado pelos profissionais de saúde e pela comunidade em geral. Além disso, por ser um instrumento aprovado pelo Conselho Municipal de Saúde, a equipe do NASF Itapema fica respaldada e garante a legitimação das suas intervenções, não estando, assim, à mercê da política de governo.

Desenvolve-se esse trabalho sobre a ótica da Profissional de Educação Física que compõe a equipe NASF Itapema e, em dado momento, será dada ênfase à organização de seu processo de trabalho. O objetivo geral é apresentar o Protocolo de Atendimento do Núcleo de Apoio à Saúde da Família de Itapema - SC. E como objetivos específicos: oportunizar para que o protocolo de atendimento NASF Itapema possa servir de embasamento para que outras equipes NASF produzam o seu próprio protocolo; e explicitar o fluxograma de atendimento do Profissional de Educação Física da equipe NASF Itapema.

Justifica-se o estudo pela não existência de protocolos de atendimento NASF disponíveis que possam embasar novas equipes e levantar possibilidades para equipes já instituídas. Reforça-se a importância de ser um documento/instrumento construído coletivamente, a partir da realidade do município de Itapema, tendo sido considerada de suma importância obter o aval da população por meio de órgão representativo, como o Conselho Municipal de Saúde.

\section{O CASO NASF}

A equipe NASF Itapema refere-se à modalidade de NASF 1, que atende de 5 a 9 Equipes de ESF e/ou de Atenção Básica, com equipe multiprofissional que totalize a carga horária mínima de 200 horas semanais, conforme Portaria no 3.124/2012, que obteve seu cadastramento junto ao Ministério da Saúde (MS) em 2008. Porém, seu efetivo funcionamento, como prevê o MS, se deu a partir de 2011, quando a coordenadora da equipe NASF, que na época era a profissional médica ginecologista/obstetra, chamou individualmente os profissionais cadastrados no Cadastro Nacional de Estabelecimentos de Saúde (CNES) para verificar se permanecia no profissional o interesse em compor a equipe, bem como pactuar a estruturação do processo de trabalho e efetiva atuação como equipe NASF. Este processo deu-se de dezembro de 2011 a março de 2012, quando a Profissional de Educação Física da Secretaria da Saúde foi convidada a integrar a equipe, finalizando sua composição.

Iniciaram-se então as reuniões para pactuar o serviço, capacitar-se e organizar os processos de trabalho individuais e coletivos. A equipe contava com oito profissionais cadastrados, 
sendo 01 fisioterapeuta, 01 fonoaudióloga, 01 médica ginecologista/obstetra, 01 nutricionista, 01 profissional de educação física, 02 psicólogas, 01 terapeuta ocupacional e, não cadastrado, 01 profissional de apoio da enfermagem. Após estas etapas, priorizaram-se as apresentações sobre o que é o NASF, quais eram os profissionais que o compunham, suas formações e especializações, horas semanais disponibilizadas, etc. Estas informações foram levadas aos profissionais da Secretaria de Municipal de Saúde que atuavam na Atenção Básica. Para tanto, foram utilizados espaços de reuniões coletivas, reuniões por categoria profissional, participação nas reuniões de equipe nas UBS e posteriores capacitações.

Em agosto de 2012, a equipe NASF passou por nova reestruturação, devido à saída de alguns profissionais da Secretaria Municipal de Saúde, inclusive da coordenadora. Atualmente, a equipe NASF optou por desenvolver uma coordenação compartilhada entre os componentes, e conta com os seguintes profissionais: 01 farmacêutica, 01 fisioterapeuta, 01 fonoaudióloga, 01 nutricionista, 01 profissional de educação física e 01 psicóloga.

O Protocolo de Atendimento do Núcleo de Apoio à Saúde da Família (PANASF) de Itapema - SC foi elaborado devido à necessidade percebida pelas profissionais que o compõem, necessidade esta advinda da importância de ter respaldo nas atuações e de organizar o processo de trabalho e a rede de atendimento. Visou-se qualificar o serviço e evitar que a política de governo impere sobre as atuações da equipe. Por meio do PANASF, objetivou-se ainda uma maior divulgação da composição da equipe NASF Itapema e facilitar a compreensão dos profissionais de saúde e população em geral acerca do que é o Núcleo de Apoio à Saúde da Família, como solicitar/ acessar este apoio e quais os serviços prestados pela equipe NASF de Itapema.

A decisão da criação do protocolo de atendimento surgiu na reunião de equipe NASF em 18 de dezembro de 2012. Sua elaboração teórica foi iniciada em janeiro de 2013 pelas profissionais de Educação Física e Nutrição, para posterior contribuição das profissionais de Psicologia, Fisioterapia, Fonoaudiologia e Farmácia. Para que se pudesse embasar, realizou-se uma pesquisa na internet sobre bibliografias, artigos e/ou relatos de experiência disponíveis para consulta que referenciassem protocolos de atendimento para a equipe NASF e obtive-se uma negativa. Selecionou-se, assim, o protocolo de Saúde Mental de Florianópolis SC de 2010, para se "espelhar" para a criação do protocolo, juntamente às informações disponibilizadas nos Cadernos de Atenção Básica - Núcleo de Apoio à Saúde da Família (Cadernos 27 e, posteriormente, o 39 dos anos 2010 e 2014 respectivamente), conhecimento do território e das equipes de cobertura sob responsabilidade da equipe NASF, somadas às construções teórico/práticas pregressas.

Em fevereiro de 2013, após finalizar a construção do PANASF, convocou-se uma reunião com a direção de atenção básica, direção de atenção especializada, assessora especializada para políticas de saúde e secretaria municipal de saúde de Itapema para que, por meio de slides, material impresso e verbalmente, pudesse apresentá-lo. Todos os presentes o aprovaram e sugeriram que fosse apresentado ao Conselho Municipal de Saúde (CMS), para posterior divulgação como documento legitimado da Secretaria Municipal de Saúde do município de Itapema. Em outubro de 2013 a Profissional de Educação Física do NASF prontificou-se a apresentá-lo no CMS, momento em que foi realizada, integralmente, sua leitura coletiva, oportunizando momentos para considerações e questionamentos dos conselheiros locais de saúde, profissionais de saúde e comunidade em geral que se fizera presente.

Após a explanação e sanadas as dúvidas, o Protocolo de Atendimento NASF foi aprovado por unanimidade. Porém, infelizmente, o mesmo não foi divulgado amplamente pela Secretaria 
Municipal de Saúde, ficando restrito ao conhecimento dos que participaram das reuniões. Tentando resgatar sua divulgação, em setembro de 2014, o mesmo foi atualizado e reapresentado pela Profissional de Educação Física no CMS, sendo aprovadas suas alterações/atualizações. Em outubro de 2014, o PANASF foi reencaminhado à direção de atenção básica para sua divulgação, e até o presente momento se permanece no aguardo de sua publicação.

No PANASF contém uma breve introdução conceituando o NASF, explanação sobre a metodologia de construção e aprovação do protocolo, pontuando que o mesmo deverá ser revisado anualmente por necessidade de ser alterado para melhor atender às necessidades que são voláteis, estando assim em constante processo de aperfeiçoamento e reconstrução.

Citam-se ainda no PANASF quais são os profissionais que atualmente compõem a equipe NASF Itapema e a sua carga horária no município, bem como as atividades desenvolvidas coletiva e individualmente por categoria profissional. Coletivamente a ser desenvolvidas, definiram-se as ações, como: reuniões de equipe NASF; participação nas reuniões de equipe das Unidades Básicas de Saúde (UBS); grupos de educação em saúde; educação permanente; interconsultas; métodos de avaliação e ações administrativas.

Foi elaborada ainda uma representação do atendimento geral dos profissionais do NASF, colocando-os em importância horizontal com relação a seus pares, não hierarquizando, reforçando que sua atuação deve dar-se multiprofissionalmente e de maneira compartilhada com as equipes das UBS, direta ou indiretamente com os usuários dos serviços de saúde, dando prioridade de atuação por meio de atividades grupais. Já os atendimentos individuais não são priorizados, sendo realizados apenas quando a equipe da UBS e/ou o profissional do NASF detecta necessidade desta conduta, não mantendo assim uma agenda aberta para tais fins.

Após a construção teórica do PANASF e a pactuação das atividades a serem desenvolvidas coletivamente, foram elaborados, individualmente, os fluxogramas de atendimento por categoria profissional, mantendo a autonomia de cada profissional.

Como recorte, a partir deste momento será dado enfoque na organização do processo de trabalho da Profissional de Educação Física, iniciando com a apresentação do fluxograma desenvolvido e considerações acerca das atividades propostas.

Figura 1: Fluxograma de atendimento: profissional de educação física

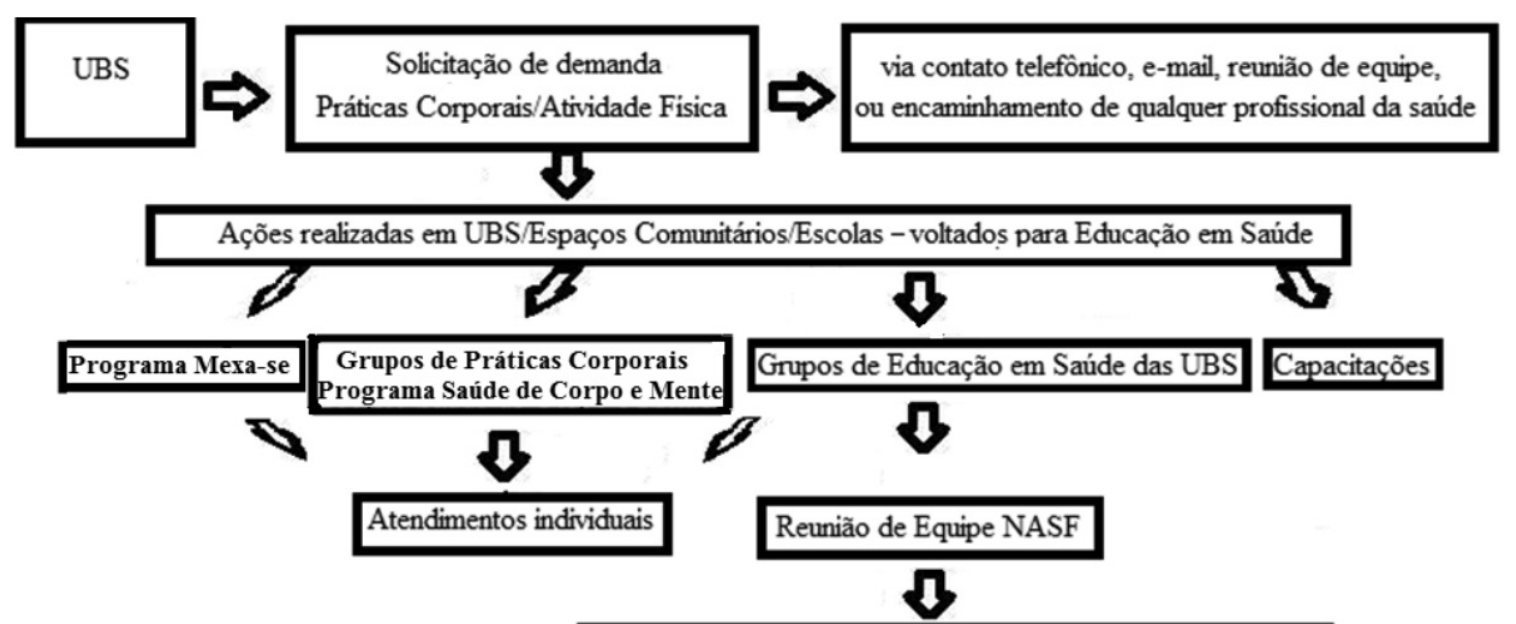

Avaliação, discussão de resultados, comentários e impressões

Fonte: Elaborada pelos autores. 
Conforme o fluxograma apresentado, a solicitação da demanda parte das equipes de UBS, que solicitam o apoio da Profissional de Educação Física por meio de contato telefônico, e-mail, pessoalmente em reuniões de equipe ou por meio de encaminhamento de qualquer profissional de saúde por escrito. O profissional recebe as solicitações, analisa e as responde positivamente, com abertura para sugestão e reestruturação da abordagem às temáticas das demandas recebidas, inclusive sugerindo a participação de outros profissionais da saúde na elaboração e na aplicação das atividades. Ou, negativamente, reagendando para outra data, sugerindo outro tema ou outro profissional para desenvolvê-lo. Quando a profissional detecta a demanda, ela sugere ações que possam ser realizadas. As ações podem ser desenvolvidas nas UBS, Espaços Comunitários e Escolas, destinadas aos profissionais de saúde e comunidade em geral com o foco na educação em saúde. Tudo o que é desenvolvido fica registrado em ata individual por categoria profissional.

Algumas das solicitações e das ações desenvolvidas passaram posteriormente a se tornar programas de atuação, permanecendo fixas nos espaços de saúde, como o Programa Mexa-se (turmas com duração de três meses), Programa Saúde de Corpo e Mente(turmas contínuas, sem término previsto), sendo os dois programas para oportunização e desenvolvimento de práticas corporais, inicialmente assistidas pela profissional, buscando proporcionar o desenvolvimento de práticas conscientes e prazerosas, que possam ser disseminadas pelos participantes com segurança, tonando-se um hábito e, por consequência, parte do estilo de vida. Os Grupos de Educação em Saúde nas UBS são fixos à medida que ocorrem, normalmente, os grupos programáticos das UBS, como Hipertensos e Diabéticos e Gestantes. Já as Capacitações para Servidores da Saúde são organizadas a partir de demanda trazida pelos profissionais (por equipe ou categoria profissional) e/ou solicitação da Secretaria Municipal de Saúde. Todos estes programas e encontros realizados são voltados à prevenção, à proteção, à recuperação e à promoção da saúde, envoltos na temática das práticas corporais.

Quando a equipe da UBS ou a própria Profissional de Educação Física detecta a necessidade de atendimento individual ou visita domiciliar, ela organiza sua agenda para tal atividade. As demandas gerais são levadas semanalmente à reunião de equipe NASF, quando são realizadas avaliações, discussão de resultados, planejamentos, comentários e impressões que ficam registradas em livro ata da equipe NASF. Quando pertinente, após a reunião de equipe NASF, a profissional dá devolutiva aos profissionais da UBS e aos usuários do serviço de saúde.

Os conceitos que permeiam a prática do Profissional de Educação Física na saúde são essencialmente o das práticas corporais e da corporeidade, devido à concepção ampliada de ser humano como ser integral dotado de intencionalidades.

A corporeidade é vista como manifestação, expressão e intencionalidade humana, sendo cada gesto significante, estando em íntima relação com o contexto social em que se vive (RAUBER; JAEGER, 2002). Para Santin (1987, p. 26), "O homem é movimento, o movimento que se torna gesto, o gesto que fala, que instaura a presença expressiva, comunicativa e criadora”, compreendendo o ser humano com um ser sujeito de suas ações, reflexivo, crítico, intencional e significativo. Segundo Rauber e Jaeger (2002), a corporeidade é o que caracteriza o humano no homem.

Assim, não poderia deixar de ser abordado este conceito que posiciona o ser humano como quem transcende a representação de corpo como puramente biológico e que tem valorizadas as suas manifestações por meio do movimento consciente e reflexivo que foge ao tecnicismo. Para tanto, o conceito de práticas corporais apresenta uma possibilidade de desvelamento a partir deste movimento, sendo a base da atuação do Profissional de Educação Física na saúde. Corroborando, Marques (1987, p. 08) assevera que: 
Mais do que ter um corpo que se usa como objeto ou como instrumento, o homem precisa ser corpo, realizando a autoconstrução corporal da consciência de si e da expressividade relacional, vivendo o corpo como trabalho e lazer, como gesto, harmonia, arte e espetáculo.

Melo, Antunes e Schneider (2005) compreendem as práticas corporais como expressões concretas de formas de educação do corpo, de descobertas e de vivência de prazeres, as quais são carregadas de valores, ideais de corpos, saúde, beleza e até mesmo de felicidade, consolidando uma proposta que busca a saúde integral ao longo do curso da vida.

Basei (2008) sustenta, ainda, que o corpo é a condição de existência do homem no mundo, e as práticas corporais são sua forma máxima de expressão de desejos, necessidades, emoções, conflitos e, por que não, de pertencimento e identificação a um determinado grupo social e sua cultura identitária.

Carmello (2002 apud BONETTI; ALARCON; BERGERO, 2005, p. 182) enfatiza que:

Uma prática corporal prazerosa permite criar condições de si, que consequentemente causará influência em suas atitudes e em sua maneira de se posicionar. Permite ainda que as pessoas se desinibam, sintam uma liberdade interior, experimentem um melhor estado de espírito e uma sensação agradável em seu corpo.

Relacionando corporeidade e práticas corporais, compreende-se que as duas sintonizamse e coexistem no movimento quando este é significante, reflexivo e respeita as individualidades, os desejos, os anseios, a história pregressa e o meio em que o praticante encontra-se e com o qual se identifica.

As práticas corporais seriam, então, perceber o ser humano além do movimento, e não o movimento por si só. Elas apresentam-se como metodologia de ressignificações das práxis deterministas estereotipadas e preponderantemente diretivas por parte dos Profissionais de Educação Física, podendo ser, ao praticante, libertadora e promotora de saúde.

\section{CONSIDERAÇÕES FINAIS}

A criação do Protocolo de Atendimento do Núcleo de Apoio à Saúde da Família de Itapema - SC surgiu a partir da necessidade de organização do serviço por parte dos profissionais que o compõem, não sendo imposto. Ele vem com a possibilidade de dar visibilidade ao trabalho da equipe NASF, pactuar o acesso e garantir ações mínimas por parte de cada categoria profissional, não excluindo a atuação coletiva em momento algum. Com este protocolo garantem-se, como dito anteriormente, ações mínimas, ficando o que não está explicitado no fluxograma a cargo da autonomia de cada profissional analisar, considerar se está qualificado e se há possibilidades de atender as demandas extras às atividades do fluxograma, não "engessando sua atuação" ou restringindo às práticas devido à existência deste.

Considera-se de fundamental importância para a organização do processo de trabalho, este sendo apoiado por protocolo ou não, que sejam valorizadas as especificidades dos profissionais de saúde e que seja garantida sua autonomia. Que se considerem as redes de apoio intra e intersetoriais, as particularidades de cada localidade, o perfil populacional, as necessidades, os anseios e as potencialidades das equipes de saúde e população em geral. Faz-se necessário ampliar 
o olhar e a escuta ativa, só assim os atendimentos, as atividades ou os grupos propostos terão adesão e serão significantes. É fundamental a valorização de saberes técnicos e populares, para que individualmente e coletivamente possa haver a reconstrução de saberes, que consequentemente refletirão na concepção de saúde e de qualidade de vida.

O fluxograma de atuação da Profissional de Educação Física do NASF Itapema contempla atividades que garantem acesso a qualquer pessoa que tenha interesse em experienciar as práticas corporais. $\mathrm{O}$ acesso pode ser iniciado por contato facilitado com o profissional, tanto pessoalmente ou por meio da equipe de saúde. Porém, na maior parte das vezes se dá por indicação de algum profissional da saúde que passa a ser disseminador e incentivador destas práticas.

Com a atuação do Profissional de Educação Física na saúde embasada na corporeidade e nas práticas corporais, permite-se que não haja exclusão de nenhum participante, pois as atividades são distintas e adaptáveis, respeitando as suas culturas de movimento, não visando à performance, mas sim bem-estar, integração e socialização, com foco de possibilitar sentimentos de pertencimento, identidade comunitária, solidariedade, prazer e promoção da saúde.

\section{REFERÊNCIAS}

BASEI, A. P. As práticas corporais na cultura escolar: a estrutura do contex to e a construção de significados. Revista Digital efdeportes. Buenos Aires - Año 13 - ํ121 - Junho de 2008. Disponível em: http:// www.efdeportes.com/. Acesso em: 13/09/209.

BRASIL. Ministério da Saúde. Secretaria de Atenção à Saúde. Departamento de Atenção Básica. Diretrizes do NASF: Núcleo de Apoio a Saúde da Família / Ministério da Saúde, Secretaria de Atenção à Saúde, Departamento de Atenção Básica. - Brasília: Ministério da Saúde, 2010. 152 p.: il. - (Série A. Normas e Manuais Técnicos) (Caderno de Atenção Básica, n. 27).

BRASIL. Ministério da Saúde. Secretaria de Atenção à Saúde. Departamento de Atenção Básica. Núcleo de Apoio à Saúde da Família / Ministério da Saúde, Secretaria de Atenção à Saúde, Departamento de Atenção Básica. - Brasília: Ministério da Saúde, 2014. 112 p.: il. - (Cadernos de Atenção Básica, n. 39).

BONETTI A.; ALARCON, M.; BERGERO, V. Re-significando práticas corporais na prevenção e reabilitação cardiovascular: In: SILVA, A. M. e DAMIANI, R. I. Práticas Corporais: Experiências em Educação Física para a outra formação humana. Nauemblu Ciência \& Arte, 2005 Florianópolis, SC Brasil. v.3 Ministério dos Esportes.

MELO, C. K.; ANTUNES, P. C.; SCHNEIDER, M. D. Desenferrujando as dobradiças: as práticas corporais na maturidade: In: SILVA, A. M. e DAMIANI, R. I. Práticas Corporais: Trilhando e compar(trilhando) as ações em Educação Física. Nauemblu Ciência \& Arte, 2005. Florianópolis, SC - Brasil. v.2. Ministério dos Esportes.

RAUBER, A.; JAEGER, A. A. "Vivemos num mundo diferente”: reflexões acerca da corporeidade das crianças e dos adolescentes em situação de rua. Revista Kinesis, Santa Maria, n² 26, p. 70-166, Maio de 2002.

SANTIN, Silvino. Educação Física: uma abordagem filosófica da corporeidade. Ijuí: UNIJUÍ, 1987. 125p. (Coleção ensaios: política e filosofia)

MARQUES, M. O. In: SANTIN, Silvino. Educação Física: uma abordagem filosófica da corporeidade. Ijuí: Liv. UNIJUÍ ED., 1987. 125p. (Coleção ensaios: política e filosofia; v.2). 
34- RBTS - v.1, n.2, 2014

SECRETARIA MUNICIPAL DE SAÚDE. Protocolo de Atenção em Saúde Mental Município de Florianópolis - Tubarão: Ed. Copiart, 2010. 